\title{
Cyclosporine in ocular surface inflammation
}

P Hossain ${ }^{1,2}$

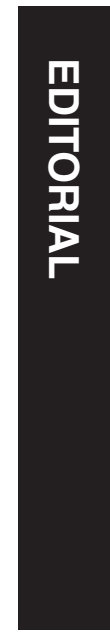

Eye (2017) 31, 665-667; doi:10.1038/eye.2017.5; published online 17 February 2017

Use in dry eye disease: influence on related corneal neuropathy?

Dry eye disease (DED) is the most frequent encountered ocular surface condition and remains one of the world's most overlooked and treatment elusive ocular conditions. ${ }^{1,2}$ There a multitude of lubricating eye drops, agents/approaches to deal with the condition and associated external eye disease. ${ }^{3}$ Without gaining much in symptomatic relief.

In 2007, the International Dry Eye WorkShop produced the DEWS report. ${ }^{1,3,4}$ Over the past decade, this has made a significant impact rationalising the approach on the management of DED. For example, the DEWS report has been particularly helpful in dividing the classification of DED into Evaporative and Aqueous Deficient Dry Eye. ${ }^{4}$ The report has also helped to focus on improving the tools for DED assessment and stimulated pharmaceutical firms to develop new therapies.

We also know there have been many weaknesses of the original DEWS report and the International Working Group have recently reconvened and a revised version DEWS report, the DEWS II report is to be published soon. ${ }^{5}$

From DEWS I, a strong role for inflammation in DED pathogenesis is described. ${ }^{4}$ Chronic inflammation leads to structural changes to the corneal surface and anti-inflammatory agents like topical steroids in varying strengths can improve symptoms. If inflammation could be controlled over the long-term, we would expect more sustained benefits in symptom control. However, the use of steroids over longer periods is limited by the risks of intraocular pressure rise and/or cataract formation. $^{3}$

An alternative to long-term topical steroids is the use of topical cyclosporine. ${ }^{6}$ Different formulations of this agent have been shown to improve features in DED with a lower risk profile than topical steroid. Topical cyclosporin can safely control chronic ocular surface inflammation over a long period. Such improved control offers the opportunity to evaluate the changes in corneal structure. ${ }^{3}$

In this issue, Iaccheri et $a l^{7}$ have used topical cyclosporine $0.05 \%$ twice a day for 6 months in patients with established dry eye disease (Sjögren's and Non- Sjögren's). The drug appeared to be well tolerated with $<5 \%$ of patients discontinuing the medication. As expected, there were improvements in DED symptoms with ocular surface parameters such as improvements in OSDI questionnaire, tear break time and ocular surface staining.

What appears more interesting are the structural changes found on corneal confocal microscopy over a 6-month period of therapy. They confirmed findings that corneal epithelial cell density substantially increased with lower 'keratocyte activation'. ${ }^{8}$ But they show the number of sub-basal nerves were reduced by almost half with similar changes to nerve reflectivity and tortuosity. The results provide the strongest evidence so far for a correlation between the changes in nerve morphology and the degree of dry eye.

Results from previous studies examining the changes to the subepithelial plexus have produced conflicting findings. Some showing decreased corneal innervation in DED, whereas others show increased corneal innervation. ${ }^{9-12}$ The controversy may be due to the kind of confocal device used or from small number of patients or due to difficulties in the sample area of examination. ${ }^{12}$ In the study by Iaccheri et al, ${ }^{7}$ serial laser scanning confocal microscopy was performed by an expert observer, doing multiple examinations in a large cohort of treatment responsive patients.

Patients with DED have hyper-regeneration of nerves at presentation, indicating that there is a pathological nerve response to the presence
${ }^{1}$ Division of Clinical and Experimental Sciences, Faculty of Medicine, University of Southampton, Southampton, UK

${ }^{2}$ University Hospital Southampton NHS Foundation Trust, Southampton, UK

Correspondence: P Hossain, Eye Unit, University of Southampton, Southampton General Hospital, Tremona Road, Southampton SO16 6YD, UK

Tel: +44 (0)23 8120 4270; Fax: +44 (0)23 81204436 . E-mail: parwez@soton.ac.uk 
of chronic DED. This suggests patient pain symptoms are partly due to a neuropathic process. Using cyclosporin for 6 months appears to change the corneal nerve features.

In this journal, we have previously highlighted how important corneal nerve changes contribute to the features of DED. ${ }^{13}$ Pain can originate from both nociceptive and neuropathic sources. DED patients often describe features of neuropathic pain, which include spontaneous pain, dysesthesias (unpleasant abnormal sensations), and hyperalgesia (exaggerated pain response to supra-threshold noxious stimuli). The discomfort can lead to the development of psychosocial problems such as depression, stress, anxiety, and sleep disorders. $^{13}$

We currently do not assess the presence of neuropathic ocular pain in DED. We often fail to consider this possibility. Now with many more treatments for DED on the horizon and the effects of more established treatments on corneal nerve features being understood. Assessing and treating corneal neuropathy in DED is likely to be more important when considering our treatment options. $^{13}$

\section{Use in adenoviral keratitis: reduction on subepithelial infiltrates?}

Adenoviral keratitis can be visually debilitating, both in the acute phase when there pseudomomembrane formation or later there is corneal involvement with subepithelial inflammatory infiltration. Treatment is largely conservative, although controversial many clinicians use topical steroids to help the recovery process to aid clearance of corneal changes. ${ }^{14}$ However, often this approach can lengthen the disease process, lead to rebound inflammation and a possible risk of increased adenovirus replication and extended viral shedding. There is the additional risk of use of topical steroids making the eye prone to intraocular pressure rise and lens opacification. As an immunosuppressant, cyclosporine, targeting CD4+ T cells, it appears rational to apply it sue to other ocular surface inflammatory conditions. However, there are fears that in infective conditions, the use of this agent could perpetuate the infective process.

In this issue, Leyla et $a l^{15}$ have shown for the first time, that topical cyclosporine $2 \%$ given in the first 3 weeks, can reduce corneal subepithelial infiltrates (SEI). They show that its use also shortens the duration of acute adenoviral symptoms.

Unlike other studies of topical cyclosporine, ${ }^{16,17}$ where the agent has been used after the onset of SEI complications. Leyla et al have used it at first clinical presentation and compared their outcomes with topical steroids. They found that symptoms were less severe with cyclosporine. However, their study was limited by only a 3 month follow-up period and not all patients escaped developing SEI. Nevertheless, a significant number had improved outcomes with cyclosporine alone. ${ }^{15}$

\section{Conflict of interest}

The author declares no conflict of interest.

\section{References}

1 [No authors listed] The epidemiology of dry eye disease: report of the Epidemiology Subcommittee of the International Dry Eye WorkShop (2007). Ocul Surf 2007; 5: 93-107.

2 Hossain P, Konstantopoulos A. Blepharitis: remains a diagnostic enigma. A role for tea tree oil shampoo? Eye (Lond) 2015; 29: 1520-1521.

3 [No authors listed] Management and therapy of dry eye disease: report of the Management and Therapy Subcommittee of the International Dry Eye WorkShop (2007). Ocul Surf 2007; 5: 163-178.

4 [No authors listed] The definition and classification of dry eye disease: report of the Definition and Classification Subcommittee of the International Dry Eye WorkShop (2007). Ocul Surf 2007; 5: 75-92.

5 DEWS II The Dry Eye Workshop 2016. Available at http:// www.tfosdewsreport.org/.

6 Sall K, Stevenson OD, Mundorf TK, Reis BL. Two multicenter, randomized studies of the efficacy and safety of cyclosporine ophthalmic emulsion in moderate to severe dry eye disease. CsA Phase 3 Study Group. Ophthalmology 2000; 107: 631-639.

7 Iaccheri B, Torroni G, Cagini C, Fiore T, Cerquaglia A, Lupidi M, Cilliona $\mathrm{S}$ et al. Corneal confocal scanning laser microscopy in patients with dry eye disease treated with topical cyclosporine. Eye (Lond) 2017; 31: 788-794.

8 Villani E, Galimberti D, Viola F, Mapelli C, Ratiglia R. The cornea in Sjogren's syndrome: an in vivo confocal study. Invest Ophthalmol Vis Sci 2007; 48: 2017-2022.

9 Benitez del Castillo J M, Wasfy M A, Fernandez C, Garcia-Sanchez J. An in vivo confocal masked study on corneal epithelium and subbasal nerves in patients with dry eye. Invest Ophthalmol Vis Sci 2004; 45: 3030-3035.

10 Tuisku IS, Konttinen YT, Konttinen LM, Tervo TM. Alterations in corneal sensitivity and nerve morphology in patients with primary Sjogren's syndrome. Exp Eye Res 2008; 86: 879-885.

11 Zhang M, Chen J, Luo L, Xiao Q, Sun M, Liu Z. Altered corneal nerves in aqueous tear deficiency viewed by in vivo confocal microscopy. Cornea 2005; 24: 818-824.

12 Villani E, Baudouin C, Efron N, Hamrah P, Kojima T, Patel SV et al. In vivo confocal microscopy of the ocular surface: from bench to bedside. Curr Eye Res 2014; 39: 213-231.

13 Galor A, Levitt RC, Felix ER, Martin ER, Sarantopoulos CD. Neuropathic ocular pain: an important yet underevaluated feature of dry eye. Eye (Lond) 2015; 29: 301-312. 
14 Wilkins M R, Khan S, Bunce C, Khawaja A, Siriwardena D, Larkin DF. A randomised placebo-controlled trial of topical steroid in presumed viral conjunctivitis. Br J Ophthalmol 2011; 95: 1299-1303.

15 Asena L, Şıngar Özdemir E, Burcu A, Ercan E, Çolak M, Altınörs DD. Comparison of clinical outcome with different treatment regimens in acute adenoviral keratoconjunctivitis. Eye (Lond) 2017; 31: 781-787.
16 Jeng $\mathrm{BH}$, Holsclaw DS. Cyclosporine A 1\% eye drops for the treatment of subepithelial infiltrates after adenoviral keratoconjunctivitis. Cornea 2011; 30: 958-961.

17 Okumus S, Coskun E, Tatar MG, Kaydu E, Yayuspayi R, Comez A et al. Cyclosporine a $0.05 \%$ eye drops for the treatment of subepithelial infiltrates after epidemic keratoconjunctivitis. BMC Ophthalmol 2012; 12: 42 . 